\title{
Enquête sur les Farines Céréalières et les Ingrédients Utilisés Dans la Préparation de la Pâte Alimentaire Traditionnelle Appelée "Tôh" à Abidjan (Côte d'Ivoire)
}

\author{
Claude Ako Memel Nomel, \\ Yao Kouadio, \\ Ghislaine Chépo Dan, \\ Thierry Komadé, \\ Patrice Lucien Kouamé,
}

Laboratoire de Biocatalyse et des Bioprocédés, UFR des Sciences et Technologies des Aliments, Université Nangui Abrogoua, Côte d'Ivoire

Doi: 10.19044/esj.2019.v15n6p508～URL:http://dx.doi.org/10.19044/esj.2019.v15n6p508

\section{Résumé}

La présente étude prospective vise à répertorier les différentes farines céréalières les plus vendues, consommées et préférées ainsi que les ingrédients qui font partie de la préparation de la pâte alimentaire traditionnelle appelée "tôh" à Abidjan. La méthodologie a consisté en une enquête à l'aide d'un questionnaire structuré auprès des points de vente tenus uniquement par les femmes et sélectionnés de façon aléatoire sur les marchés de trois communes du district d'Abidjan : Abobo, Koumassi et Yopougon. Ces communes ont été choisies du fait de leur forte concentration en populations allogènes du nord de la Côte d'Ivoire et des non-ivoiriens dont les céréales constituent la base alimentaire. Il en résulte de l'enquête que 5129 points de vente ont été recensés. Les farines de céréales les plus vendues, utilisées pour la préparation du "tôh" et consommées sont successivement les farines de maïs blanc $(36,11 \%)$, de maïs jaune $(35,18 \%)$ et de mil $(29,50 \%)$. La farine de maïs blanc est préférée à la farine de maïs jaune par les chefs de ménage de sexe masculin que de sexe féminin. Le maïs est la céréale relativement moins chère, disponible et la plus consommée chez les ménages à faible revenu (< $100.000 \mathrm{~F}$ CFA) tandis que les ménages à revenu élevé (>200.000 F CFA) préfèrent le mil. Concernant les enrichissements des farines, l'additif fréquemment utilisé pour des "tôh" plus acceptables est la potasse alimentaire artisanale $(75,35 \%)$. Cependant, les vendeuses sur les marchés devraient observer un peu plus de mesures d'hygiène aux points de stockage. 
Mots-clés: Farines de céréales, "tôh", maïs blanc, maïs jaune, mil

\title{
Survey of Cereal Flours and Ingredients Used in the Preparation of the Traditional Food Dough Called "Toh" in Abidjan (Côte d'Ivoire)
}

\author{
Claude Ako Memel Nomel, \\ Yao Kouadio, \\ Ghislaine Chépo Dan, \\ Thierry Komadé, \\ Patrice Lucien Kouamé,
}

Laboratoire de Biocatalyse et des Bioprocédés, UFR des Sciences et Technologies des Aliments, Université Nangui Abrogoua, Côte d'Ivoire

\begin{abstract}
This paper focuses on listing the various cereal flours that are the most sold, consumed and preferred, as well as the ingredients used in the preparation of the traditional pasta called "tôh" in Abidjan. The methodology consisted of a survey using a structured questionnaire at points of sale held only by women. The survey sample was randomly selected in the markets of three communes of the Abidjan district: Abobo, Koumassi, and Yopougon. These communes were chosen because of their high concentration of nonindigenous populations in northern Côte d'Ivoire and non-Ivorians whose cereals constitute the food base. As a result of the survey, 5129 outlets were identified. The best-selling cereal flours, used for the preparation of "tôh" and consumed, are successively flours of white corn $(36.11 \%)$, yellow corn $(35.18 \%)$, and millet $(29.50 \%)$. White corn flour is preferred to yellow cornmeal by male and female household heads. Maize is the relatively cheaper, available, and most consumed cereal in low-income households (<100,000 CFA). On the other hand, high-income households (> 200,000 CFA) prefer millet. Regarding enrichments of flours, the additive frequently used for "tôh" which is more acceptable is the potash food artisanal (75.35\%). However, vendors on the markets should observe more hygiene at storage points.
\end{abstract}

Keywords: Cereal flours, "tôh", white corn, yellow corn, millet 


\section{Introduction}

Les céréales sont des plantes de la famille des Poacées cultivées principalement pour leurs grains riches en amidon et pour leurs fruits récoltés dans toutes les régions du monde (Doebley et al., 1980). Dans l'espace de la Communauté des États de l'Afrique de l'Ouest (CEDEAO), l'importation des céréales représentait entre 2007-2008 une facture annuelle de 3,383 milliards de dollars, soit plus de $40 \%$ des importations agro-alimentaires (Guyomard, 2008). De ce fait, la promotion des céréales locales constitue un enjeu majeur en Afrique de l'Ouest car sa demande a évolué avec l'augmentation très rapide de la population (Bricas, 2011). En Côte d'Ivoire, les systèmes céréaliers sont centrés sur le riz (Oryzasativa L.), le maïs (Zea mays), le mil (Pennisetum glaucum) et le sorgho (Sorghum bicolor) (Beninga et al., 1990). En effet, les céréales constituent non seulement la base traditionnelle de l'alimentation mais aussi un aliment de soudure important chez les populations du nord (Sénoufo, Malinké et Mahouka) et les populations étrangères émigrées des pays d'Afrique Sud-Sahélienne (Bamba, 1985); (Beninga et al., 1990). Le mil et le sorgho, céréales parfois qualifiées de «culture du pauvre» résistent à la sécheresse des régions arides et représentent l'une des principales sources d'apport nutritionnel, tant sur les plans protéique et vitaminique que minéral (FAO, 1995) ; (FAO, 2004). Ces céréales entrent dans la composition de nombreux plats de base, dont des bouillies, des galettes et des pâtes. Parmi les pâtes de fabrication traditionnelle à base de farines de céréales se trouve le "tôh" (O'déyé, 1985). C'est un mets qui s'adapte à l'évolution du mode de vie et du monde du travail, ou le temps consacré à sa préparation et à sa consommation est court (Escalon et al., 2009). Les farines qui servent à la préparation du "tôh" sont généralement celles du maïs, du mil et du sorgho (Konkobo, 1999). Selon Konkobo (1999), le "tôh" se consomme, de préférence, avec de la sauce aux ingrédients alimentaires tels que la poudre de gombo séché, la poudre de baobab (Adansonia digitata) et le "soumbala" (ou graines de néré (Parkia biglobosa) fermentées). Sa préparation nécessite du jus de citron (Citrus), du gingembre (Zingiber), d'autres aliments et surtout de la potasse artisanale alimentaire ajoutés à la farine dont les proportions utilisées n'ont jamais été connues avec exactitude. Quelles sont donc les proportions exactes de potasse artisanale alimentaire qu'il faut pour avoir un "tôh" de bonne qualité ? Ces proportions varient- elles avec le type de céréale ? C'est pour répondre à ces préoccupations, qu'une enquête portant sur les différentes farines de céréales commercialisées et les ingrédients utilisés dans la préparation du "tôh" a été effectuée dans trois communes d'Abidjan (Côte d'Ivoire). 


\section{Méthodes}

\section{Milieu d'étude}

L'enquête a été effectuée dans trois communes du district d'Abidjan (Yopougon, Koumassi et Abobo) du 18 janvier au 3 avril des années 2008, 2009 et 2010. La collecte des données a eu lieu aux marchés des quartiers «Samanké», «Anonkoi 3» et «Sicogi» de la commune d'Abobo. Dans la commune de Yopougon, les marchés des quartiers «Sideci», «Wassakara» et «Niangon nord» ont été retenus. Quant à la commune de Koumassi, les marchés des quartiers visités sont «Djê Konan», «Progrès» et «Sicogi». Le choix de ces communes a reposé sur la forte présence des populations du nord de la Côte d'Ivoire et des non - ivoiriens dont les céréales constituent la base alimentaire.

\section{Procédure de l'enquête}

Un questionnaire structuré a permis de réaliser l'enquête. Il s'est agi de recueillir des informations sur :

- les céréales les plus vendues et consommées;

- les céréales préférées en Côte d'Ivoire ;

- les ingrédients entrant dans la production de ces farines et du "tôh".

Les questions posées étaient du type fermé (réponse par "oui" ou "non") et ouvert (donnant la latitude à la personne interrogée d'exprimer son point de vue).

\section{Traitement et analyses statistiques}

Les analyses statistiques des données ont été effectuées à l'aide des logiciels STATISTICA 7 (Stats oft Inc, Tulsa-USA Headquarters) et XLSTAT-Pro 7.5.2 (Addinsoft Sarl, Paris-France). L'effet du facteur (type de céréales) sur les variables quantitatives (paramètres étudiés) a été mis en évidence par l'analyse de variance (ANOVA) à un facteur. Les comparaisons de moyennes ont été ensuite déterminées à l'aide du test de Duncan au seuil de $5 \%$.

\section{Résultats}

Nombre de points de vente et types de farines de céréales vendues dans les communes d'Abobo, de Koumassi et de Yopougon

Un total de 5129 points de vente a été répertorié et a permis de recenser trois (3) types de farines de céréales majoritairement vendus. Ce sont les farines de maïs blanc, de maïs jaune et de mil suivies des farines de riz et de sorgho blanc. La commune d'Abobo détient 1814 (35,37 \%) de l'ensemble des points de vente enquêtés. Les communes de Koumassi et de Yopougon en possèdent respectivement $1803(35,15 \%)$ et $1512(29,48 \%)$ (Figure 1). Les pourcentages des points de vente des farines de céréales des communes 
d'Abobo et de Koumassi sont statistiquement $(\mathrm{P}>0,05)$ égaux. Par contre, ils sont significativement $(\mathrm{P}<0,05)$ supérieurs à celui de la commune de Yopougon.

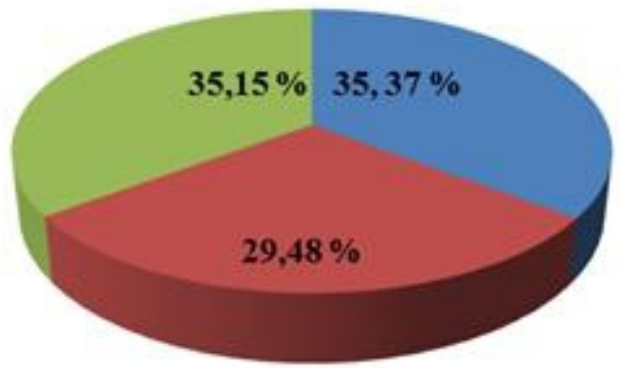

Farines recensées

Commune-Abobo $=$ Commune-Yopougon $\cong$ Commune-Koumassi

Figure 1. Proportions des points de vente des farines de céréales dans les communes d'Abobo, de Koumassi et de Yopougon

Représentativité des trois types de farines de céréales (maïs blanc, maïs jaune et mil) dans les points de vente recensés dans les communes d'Abobo, de Koumassi et de Yopougon

Sur 1814 points de vente de farine de céréales recensés dans la commune d'Abobo, 1643 (90,57 \%) concernent les farines de maïs blanc et $1621(89,36 \%)$ les farines de maïs jaune. La farine de mil est vendue sur 1550 points, soit 85,44\% de représentativité. Dans la commune de Koumassi, sur un effectif de 1803 points de vente, la farine de maïs blanc est vendue dans $1699(94,23 \%)$ points de représentativité contre $65,55 \%$ pour la farine de mil. Au niveau de la commune de Yopougon, la farine de maïs blanc détient le pourcentage de représentativité le plus élevé $(78,24 \%)$ suivie de celle de maïs jaune $(76,45 \%)$. La farine de mil a présenté le pourcentage de représentativité le moins élevé (73,08\%) (Figure 2).

Les taux des farines de céréales recensées diffèrent relativement d'une céréale à une autre. Dans toutes les communes étudiées, le maïs blanc a donné le pourcentage de représentativité le plus élevé tandis que le mil a le taux de représentativité le moins élevé. Par ailleurs, l'analyse relative au test du $\mathrm{Khi}^{2}$ au seuil de $0,5 \%$ a révélé l'existence d'une relation entre les farines de céréales recensées et les communes. 


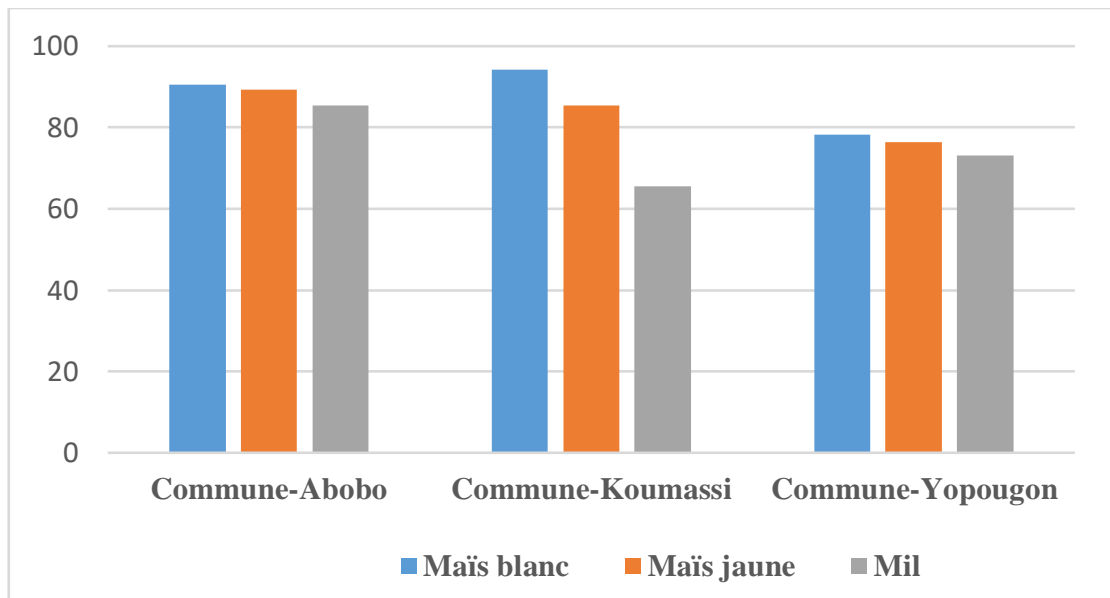

Figure 2. Représentativité des trois (3) types de farines de céréales dans les points de vente recensés dans les communes

Types et taux d'utilisation des récipients de vente des farines de céréales sur les marchés d'Abobo, de Koumassi et de Yopougon

Les commerçantes des marchés des trois communes (Abobo, Koumassi et Yopougon) conditionnent leurs différentes farines de céréales dans des récipients en aluminium majoritairement et en plastique. Sur 5219 points de vente recensés, $1376(79,94 \%)$ utilisent des récipients en aluminium pendant que $3453(20,06 \%)$ emploient des récipients en plastique (Figure 3).

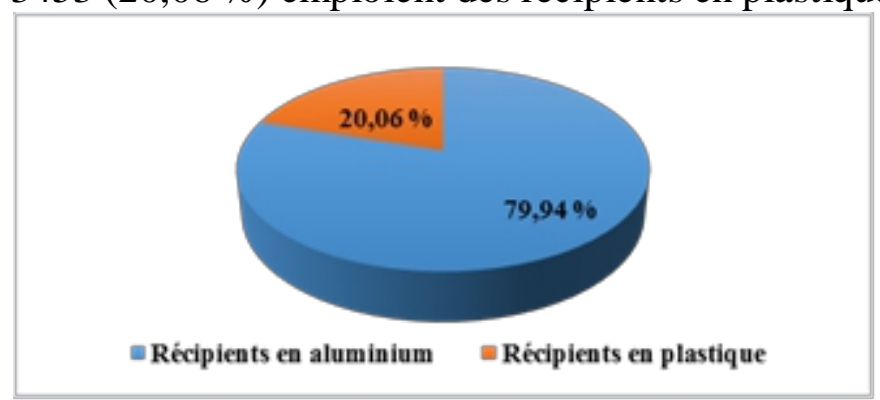

Figure 3. Types et taux d'utilisation des récipients de vente des farines de céréales sur les marchés

\section{Lieux de stockage des farines de céréales après la vente quotidienne sur les marchés d'Abobo, Koumassi et Yopougon}

Les lieux de stockage des farines de céréales après la vente quotidienne sur les marchés d'Abobo, de Koumassi et de Yopougon sont sur la table de vente au marché, au magasin de stockage ou au domicile de la vendeuse (Figure 4). Sur 5219 points de vente, 67,39 \% des commerçantes ont déclaré stocker leurs farines sur la table de vente au marché contre $12,80 \%$ de stockage dans les magasins du marché. Les vendeuses qui stockent leurs farines de céréales à leur domicile sont au nombre de 1034 (19,81\%). 


\section{8}

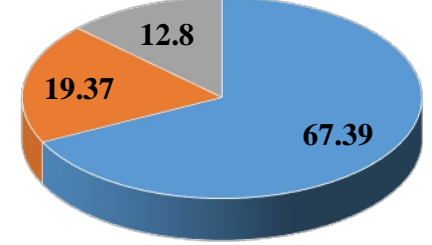

- Table de vente au marché

- Domicile de la vendeuse

\section{- Magasin de stockage du marché}

Figure 4. Lieux de stockage des farines de céréales après la vente quotidienne sur les marchés d'Abobo, Koumassi et Yopougon

Types et taux d'utilisation d'ingrédients dans la production des farines de céréales destinées à la préparation du « tôh »

Un effectif de 3865 sur 5129 personnes interrogées a reconnu que la potasse artisanale est l'un des additifs les plus utilisés dans la production des farines de céréales destinées à la confection du «tôh »; ce qui correspond à un pourcentage de représentativité de 75,35\%. Le gingembre (Zingiber) est utilisé à hauteur de 13,23\%, le citron (Citrus) à 5,79\%, le tamarin (Tamarindus indica) à $4 \%$ et le poivre (Piper) à 1,5\% (Figure 5). Le taux d'utilisation de la potasse artisanale dans la confection des farines de maïs blanc est de 19,64\% (759/3865) et de 10,10\% (390/3865) dans les farines de maïs jaune. Ce taux est à hauteur de $8,72 \%$ dans les farines de mil.

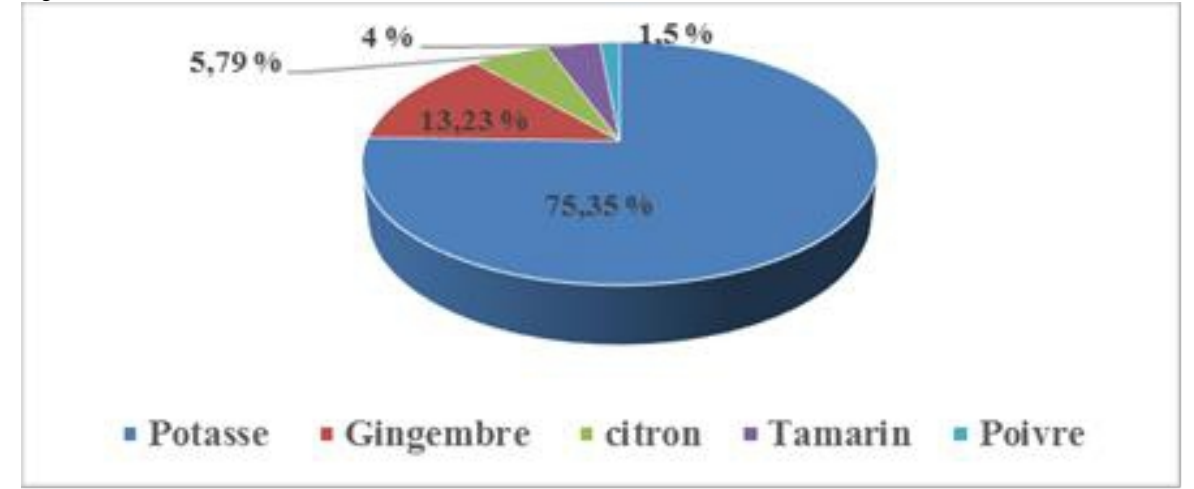

Figure 5. Taux d'utilisation d'ingrédients dans les farines de céréales destinées à la préparation du «tôh »

Taux des farines sans potasse en fonction du type de céréales dans les communes d'Abobo, de Koumassi et de Yopougon

Sur l'effectif de 5129 personnes interrogées, il est à noter que 769 $(19,89 \%)$ d'une part et $1200(31,04 \%)$ personnes d'autre part, n'utilisent pas la potasse artisanale respectivement dans les farines de maïs blanc et de mil destinées à la confection du «tôh ». Concernant les farines de maïs jaune, le 
taux de non-utilisation de la potasse est de 24,65\% (Figure 6). En outre, le test de $\mathrm{Khi}^{2}$ au seuil de $0,5 \%$ a révélé l'existence d'une relation entre l'utilisation de la potasse dans les farines de céréales et le type de céréales.

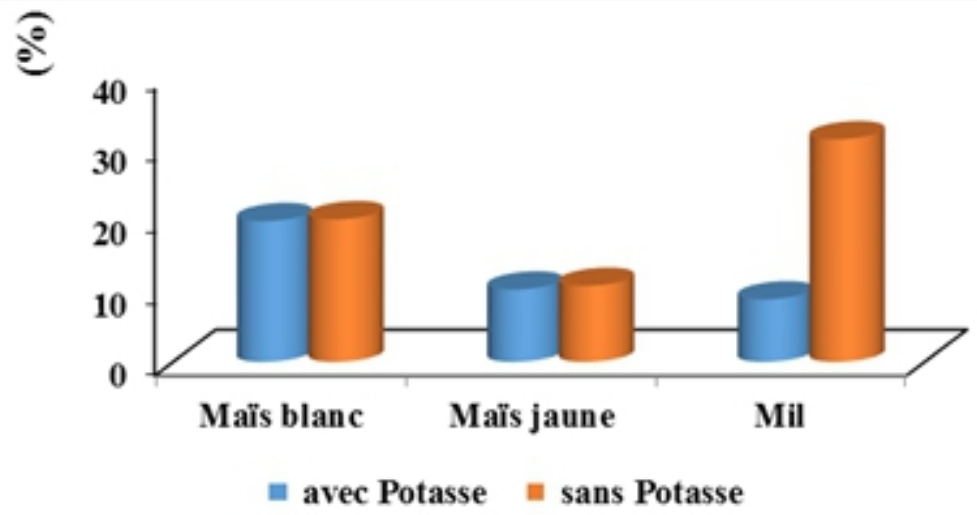

Figure 6. Taux d'utilisation des farines avec ou sans potasse en fonction du type de céréales

Taux de consommation des farines de céréales en fonction du type de céréales dans l'ensemble des communes d'Abobo, Koumassi et Yopougon

Les farines des maïs blanc et jaune ont des taux de consommation presque identiques; ils sont respectivement de $36,11 \%$ et de $35,18 \%$. Ces taux sont supérieurs à celui du mil qui est de 29,5\% (Figure 7).

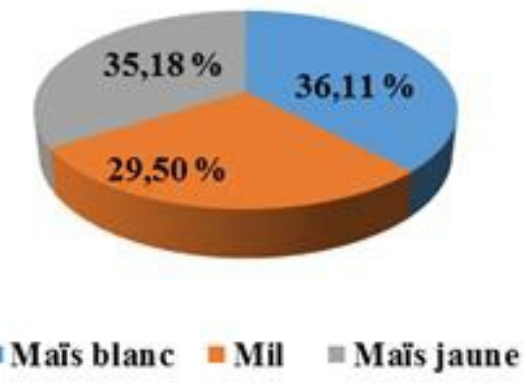

Figure 7. Répartition des taux de consommation des farines selon le type de céréales dans l'ensemble des communes

\section{Taux de préférence des farines en fonction du type de céréales et du niveau d'instruction}

Quel que soit le type de céréales, les taux de préférence des farines varient selon le niveau d'étude du chef de ménage (Figure 8). Les ménages dont les chefs sont analphabètes préfèrent la farine de maïs blanc, ensuite la farine du maïs jaune suivie de la farine du mil dans l'élaboration de leur «tôh ». Pour les chefs de ménage dont les niveaux d'étude sont le cycle primaire, les taux de préférence ne varient pratiquement pas d'une céréale à 
une autre. À partir du cycle secondaire, la farine de mil devient la plus préférée et la farine de maïs jaune est la moins appréciée. Par ailleurs, le test de Khi² au seuil de $5 \%$ a révélé l'existence d'une relation entre le type de céréales et les niveaux d'instruction.

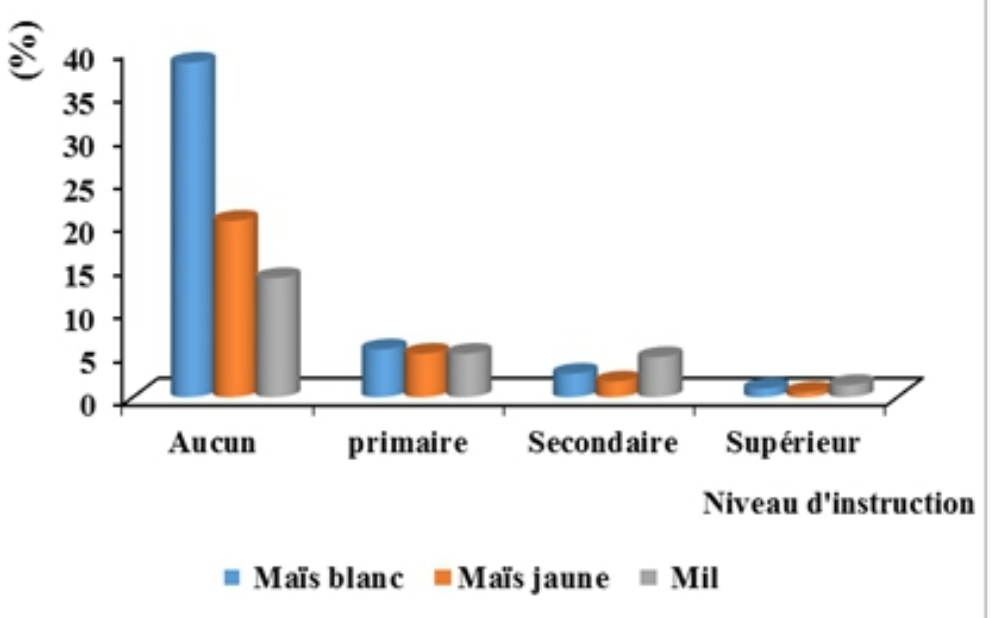

Figure 8. Taux de préférence des farines selon le type de céréales et le niveau d'étude du chef de ménage

\section{Taux de préférence des farines de céréales en fonction du type de céréales et du sexe du chef de ménage}

Les taux de préférence des farines de céréales diffèrent selon le sexe du chef de ménage quel que soit le type de céréales (Figure 9). La farine de maïs blanc est préférée par les chefs de ménage de sexe masculin $(29,2 \%)$ par rapport à ceux de sexe féminin (18,5\%). Quant aux farines de maïs jaune et de mil, un comportement contraire a été obtenu. Le test de $\mathrm{Khi}^{2}$ a révélé qu'il n'y a pas de relation entre le sexe féminin et le type de céréales. En revanche, il existe un lien entre le sexe masculin et le type de céréales.

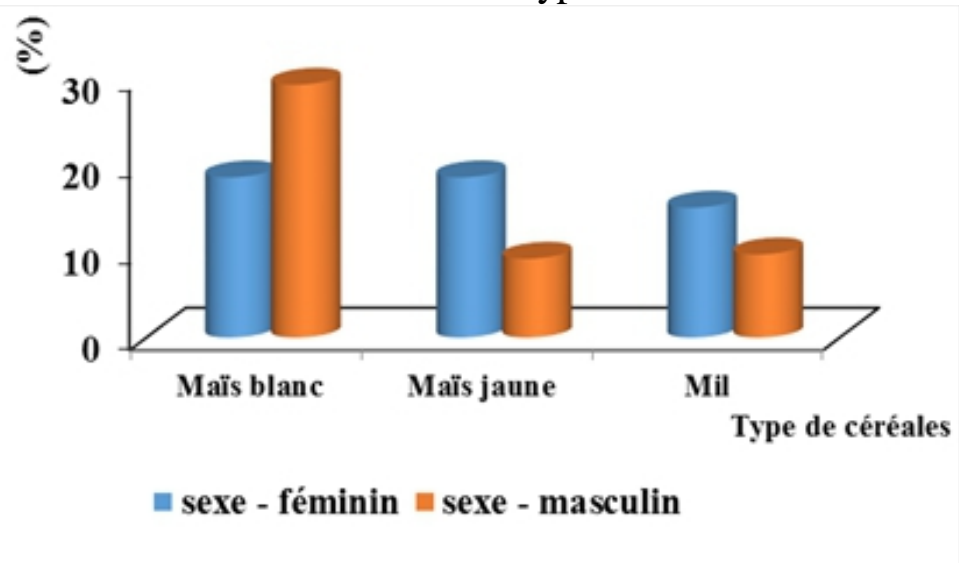

Figure 9. Taux de préférence des farines selon le type de céréales et le sexe du chef de ménage 
Taux de préférence des farines selon le revenu mensuel du chef de ménage et du type de céréales dans les communes d'Abobo, de Koumassi et Yopougon du district d'Abidjan

Les taux de préférence des farines de céréales diffèrent tant au niveau du type de farines que du revenu mensuel du chef de ménage (Figure 10). Les ménages dont le revenu mensuel est inférieur à 100.000 F CFA préfèrent les farines du maïs blanc par rapport à celles du maïs jaune et du mil. Pour les chefs de ménage dont les revenus mensuels varient entre 100 et $200.000 \mathrm{~F}$ CFA, les taux de préférence ne varient pas significativement d'une céréale à une autre. À partir des revenus mensuels supérieurs à 200.000 F CFA, la farine de mil devient la plus prisée devant celles des maïs jaune et blanc. Par ailleurs, l'analyse statistique au test $\mathrm{du} \mathrm{Khi}^{2}$ a révélé l'existence d'un lien entre le revenu mensuel du chef de ménage et le type de céréales.

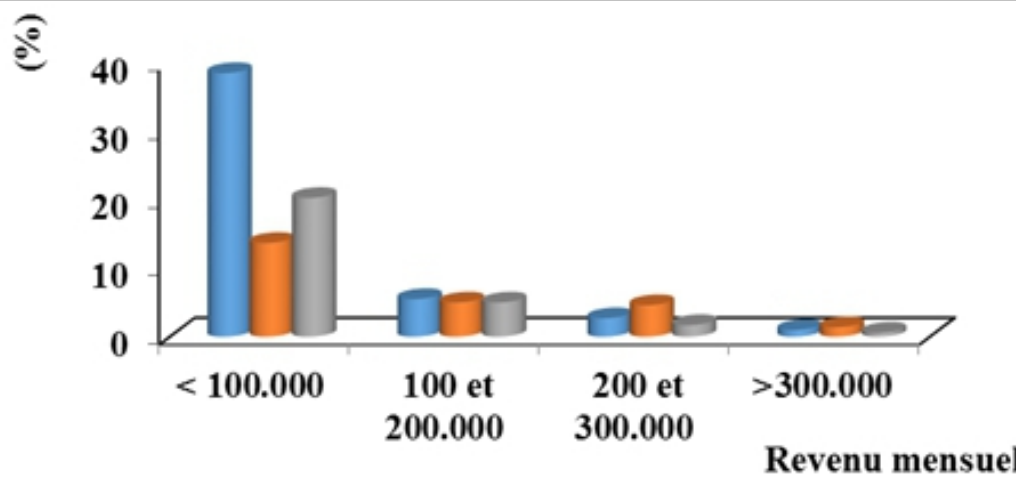

= Maïs blanc $=$ Mil $\approx$ Maïs jaune

Figure 10. Taux de préférence des types de farines de céréales selon le revenu mensuel du chef de ménage

\section{Discussion}

Les résultats relatifs aux céréales indiquent que les farines de maïs blanc sont plus commercialisées et plus consommées $(36,11 \%)$ que celles de maïs jaune $(35,18 \%)$ et de mil $(29,50 \%)$ dans l'ensemble des communes d'Abobo, de Koumassi et de Yopougon du district d'Abidjan (Côte d'Ivoire) enquêté. À l'échelle nationale, le maïs est une céréale cultivée sur tout le territoire ivoirien. Une étude menée par l'INS en 2008 a montré que 57,5\% des quantités de maïs consommées par les ménages proviendraient principalement de leur propre production contre 35,5\% qui serait à l'achat comme principale source de provenance (Elwert, 2002) ; (INS, 2008) ; (Ministère de l'agriculture, 2009). Il est disponible et relativement moins cher (Daniel et al., 2008). 
Dans la diversité de ses formes de consommation, la farine fermentée de maïs blanc est privilégiée pour la préparation du "tôh" parce qu'elle est relativement plus facile à cuisiner et moins coûteuse que celle de mil. Elle a une couleur plus blanchâtre. En effet, consommer un "tôh" blanc est devenu signe de modernité, voire de richesse comme le confirme Mitchikpe et al. (2001) et Devatour et al. (2002). Cette tendance a été observée au Burkina Faso par Diawara et al. (2002) où les céréales sont au cœur de l'alimentation. La forte présence de la farine de maïs blanc dans la répartition de la consommation et de la commercialisation des céréales s'expliquerait par les préférences et les habitudes alimentaires qui sont parfois influencées par sa disponibilité et la diversité des formes d'utilisation dont il bénéficie (Diawara et al., 2002; Daniel et al., 2008). En effet, si la farine de maïs blanc est la plus prisée, cela serait lié aux avantages qu'elle offre grâce à ses caractéristiques organoleptiques, sa qualité physique et sa commodité de transformation qui se caractérisent par la diversité de ses formes de consommation (épis frais, bouilli ou grillé, couscous, bouillie de maïs, et "tôh"). L'une de ces formes pourrait être consommée par tous les groupes ethnolinguistiques (Gutierrez, 2000) ; (Fournier et al., 2001).

Concernant la farine de mil, les taux de commercialisation et de consommation sont relativement plus faibles. Sur le plan national, très peu de ménages $(7,9 \%)$ consommeraient le mil au cours de la période 2004-2008 (INS, 2008). Le principal mode d'acquisition du mil reste l'achat (58,9\% des ménages), ce qui rendrait les coûts relativement élevés (Coates, 2007) ; (DSRP, 2009). La faible répartition de la farine de mil constatée pourrait aussi s'expliquer par le fait que le mil rentre dans la fabrication de la bière traditionnelle (tchapalo) qui a une forte valeur sociale dans les zones de savane (au nord de la Côte d'Ivoire), ce qui pourrait le rendre peu disponible sur le marché national (Seignobos, 2005) ; (Seguy, 2006).

$\mathrm{Au}$ cours de la préparation du "tôh" plusieurs ingrédients (du poivre, du piment, de la potasse, du citron, etc...) y sont ajoutés. Parmi ceux-ci, la potasse est la plus utilisée $(75,35 \%)$. En effet, la potasse améliore la texture du "tôh" en la rendant plus tendre, plus molle et plus digeste. S'agissant des communes, Abobo et Koumassi (Abidjan, Côte d'Ivoire) disposent des taux les plus élevés de farines de maïs (blanc et jaune) avec potasse. Ce constat pourrait s'expliquer par le fait que ce sont des communes cosmopolites qui auraient bénéficié de la présence migratoire des peuples, du nord de la Côte d'Ivoire, culturellement liés aux céréales et du brassage interethnique avec ceux du sud sédentaires (Courade et al., 1988). Par contre, dans la commune de Yopougon, la proportion des farines de céréales avec potasse est moindre parce qu'il y aurait une faible présence des peuples et de groupes ethniques où les céréales ne sont pas au cœur de leur alimentation. 
Les facteurs qui influencent la consommation des farines de céréales sont essentiellement les préférences alimentaires qui sont parfois conditionnées par la disponibilité de ces produits (PAM, 2008). Ces préférences varient aussi selon le niveau d'instruction, le sexe et le revenu du chef de ménage. Concernant le revenu du chef de ménage et de son niveau d'instruction, les résultats indiquent que la consommation des farines est élevée chez les chefs de ménages qui n'ont aucun niveau d'instruction et dont le revenu mensuel est moins de 100.000 F CFA. Cette observation serait due aux comportements et habitudes alimentaires mais essentiellement au pouvoir financier du chef de ménage. Selon Pierru (2008), la première différence sociale en matière d'alimentation serait de nature financière. De ce fait, plus les ménages sont-ils pauvres, plus la part de leur budget consacrée à l'alimentation en céréales est élevée (Engel, 1895). Les résultats obtenus sont en accord avec ceux de Paugams (2002) qui a observé une relation similaire entre le niveau d'instruction et le revenu du chef de ménage. Cet auteur a aussi constaté que les ménages dont la part de budget affectée aux céréales est la plus forte, sont ceux dont la personne de référence a un niveau d'études inférieur au Certificat d'Études Primaires (ouvriers, agriculteurs, employés ou sans activité professionnelle, etc...) (Paugams, 2002). Ce constat a également été fait par Caillavet (2001) qui a montré que près de $90 \%$ des ménages (à bas revenu) interrogés consacrent au moins $60 \%$ de leur budget alimentaire à la consommation des céréales. Par ailleurs, le taux de consommation des farines de céréales diminue fortement lorsque le niveau d'instruction est élevé. Cette diminution pourrait s'expliquer par la théorie inverse développée par. Ainsi, plus les ménages ont-ils un revenu élevé, moins la part de leur budget consacrée à l'alimentation des céréales est élevée (Engel, 1895).

Selon le sexe du chef de ménage, la consommation des farines de céréales varie. Les ménages à faible revenu mensuel dirigés par les femmes qui n'ont aucun niveau d'instruction, consacrent un budget alimentaire orienté davantage sur la consommation de céréales avec 43,3\% contre $29,2 \%$ chez les ménages dirigés par les hommes. Cette situation relative aux ménages à faible revenu dirigés par les femmes serait liée à des caractéristiques sociodémographiques spécifiques telles que le statut matrimonial (veuve), la taille moyenne du ménage (nombre d'enfants) et une insertion sur le marché du travail qui n'est pas toujours facile pour les femmes (Paugams, 2002) ; (Larmet, 2002). Nos résultats sont en accord avec ceux d'Andrieu (2004) qui a montré que 38,4\% du budget alimentaire des ménages pauvres sont dirigés par une femme (Andrieu, 2004).

Les farines de céréales commercialisées dans les trois communes (Abobo, Koumassi et Yopougon) sont pour la plupart conditionnées dans des récipients de vente en aluminium $(72,85 \%)$ ou en matières plastiques $(28,55$ $\%)$. L'usage en nombre important des récipients de vente en aluminium 
pourrait s'expliquer par le fait qu'ils sont obtenus, en grande partie, de fabrication artisanale, accessibles et à moindre coût. Ils sont également légers et plus résistants à la corrosion. Par contre, les récipients de vente en plastiques sont fabriqués de façon industrielle et seraient plus lourds et relativement chers (INS, 2008) ; (Chevaillet, 2009).

La table de la vendeuse $(67,39 \%)$ et son domicile $(19,37 \%)$ constituent les lieux privilégiés de la commercialisation et du stockage des farines de céréales. Cette option pourrait être dû au coût souvent élevé des magasins de stockage du marché et hors bourse des vendeuses.

Cependant, selon certains auteurs, les récipients de vente et de stockage des farines de céréales (en aluminium ou en plastiques) seraient parfois dommageables pour la santé des consommateurs. Des études ont montré que certains éléments présents ou constitutifs de ces récipients pourraient migrer dans les denrées alimentaires : cas du bisphénol-A (Barles, 2005) ; (Ngnikam et al., 2006). Aussi, les récipients (en aluminium ou en plastiques) sont-ils favorables à une humidité non contrôlée pour les farines de céréales, ce qui peut augmenter la présence de certains agents chimiques et biologiques. Les agents chimiques à risque sont essentiellement les métaux lourds, les résidus d'insecticides et des dioxines. Les agents biologiques sont souvent les insectes et les acariens, les rongeurs et/ou leurs traces macroscopiques, les moisissures, les mycotoxines, les bactéries pathogènes (salmonelles, Bacillus cereus) et d'autres impuretés (Multon, 1982). Dans tous les cas les conditions hygiéniques de stockage et de vente sont à déplorer (Chevaillet, 2009) ; (Duval, 2009).

\section{Conclusion}

Parmi les ingrédients utilisés dans les farines de céréales vendues dans les communes d'Abobo, de Koumassi et de Yopougon du district d'Abidjan (Côte d'Ivoire) pour la préparation du "tôh", la potasse artisanale alimentaire dispose du taux d'utilisation le plus élevé. Le taux d'utilisation de la potasse dans la farine de maïs blanc $(36,11 \%)$ est le plus élevé suivi de celui de la farine de maïs jaune $(35,18 \%)$ contre $(29,50 \%)$ dans la farine de mil, le moins élevé. En revanche, sans potasse, la farine de mil est la plus utilisée pour la préparation du "tôh" quelle que soit la commune.

La répartition des taux de consommation des farines de céréales en fonction du revenu mensuel, du niveau d'instruction et du sexe du chef de ménage indique que cette consommation baisse au fur et à mesure que le niveau d'instruction augmente de même que le revenu mensuel quel que soit le sexe. Il ressort de cette étude que le maïs blanc qui est la céréale relativement moins chère et disponible, est la plus consommée chez les ménages à faible revenu. Les ménages à revenu élevé, ont une préférence pour le mil. En outre, 
l'analyse relative aux résultats montre que la consommation des céréales est plus élevée chez les ménages dirigés par les femmes.

La répartition de l'utilisation de la farine de céréales en fonction des récipients de vente et des lieux de stockage révèlent que les vendeuses préfèrent les récipients en aluminium. Par ailleurs, les farines de maïs blanc et jaune les plus prisées sont commercialisées sur les tables de vente et stockées en ces lieux. Il est à noter que les conditions hygiéniques de stockage et de commercialisation des farines de céréales sont déplorables. Cette situation est favorable à la prolifération des microorganismes pathogènes et à des risques d'exposition chimique aux niveaux alimentaire et sanitaire.

\section{References:}

1. Andrieu, E. \& Caillavet, F. (2004). "Les inégalités de la consommation alimentaire en France : disparités socio-économiques et intrafamiliales du statut nutritionnel », in Caillavet F. (coord.), L'Alimentation comme dimension spécifique de la pauvreté en France, vol. 2, rapport pour l'Observatoire national de la pauvreté et de l'exclusion sociale, $197 \mathrm{p}$.

2. Bamba (1985). Impact des pistes rurales du projet Nord-est sur l'évolution des systèmes de production Lobi : cas de la sous-préfecture de Bouna. Mémoire pour un diplôme d'ingénieur agronome, Institut National Agronomique Paris-Grignon, Chaire d'agriculture comparée et de développement agricole ; Bouaké, Côte d'Ivoire, CIDT ; 104 p.

3. Barles, S. (2005). «L'invention des déchets urbains : France 1790$1970 »$, édition champ vallon, 2005.

4. Beninga, M.B., Assamoi, A.F. \& Kone, L. (1990). La prospection du sorgho. Bouaké, Côte d'Ivoire, IDESSA/DCV, 7 p.

5. Botta, H., Berdier, C. \& Deleuil, J-M. (2002). « Enjeux de la propreté urbaine », édition Presse polytechniques et universitaires romandes.

6. Bricas, N. \& Cheyns, E. (2002). La construction de la qualité comme angle d'analyse des interactions entre consommation alimentaire et savoir-faire agro-alimentaires ; Études de cas autour des produits alimentaires sur le marché de Ouagadougou.

7. Bricas, N. (2011). Suivi des facteurs de risques de crise alimentaire. Cirad. cirad.fr/actualites http://www. défis sud n 100- Bimestriel.

8. Caillavet, F., Combris, P. \& Perchard, S. (2001). L'alimentation des ménages à bas revenu, Rapport pour le CERIN (Centre de Recherche et d'Information Nutritionnelles), CORELA, $40 \mathrm{p}$.

9. Chevaillet, E. (2009). «10 questions sur les déchets en île de France », édition TECHNIP, Paris, 2009.

10. Coates, J., Swindale, A., \& Bilinsky, P. (2007). Echelle de l'Accès déterminant l'Insécurité alimentaire des Ménages (HFIAS) pour la 
Mesure de l'Accès alimentaire des Ménages : Guide d'Indicateurs. Food and Nutrition Technical Assistance Project (FANTA), Washington DC, Août 2007.

11. Courade, M. \& Droy, H. (1988). Evaluation des habitudes à la consommation des produits alimentaires en Côte d'Ivoire : Tome I, Synthèse, résumé, recommandations. Tomme II : rapport principal et annexes-Paris : ORSTOM-MSA, (rapport à la CCE-DG VIII), février 1988-86- 123p. multigr.

12. Daniel, M. \& Richard, C. (2008). “Coping Strategies Index”, USAID, CARE, WFP, FIC, TANGO.

13. Devatour, H., Moity-Maizi, P. \& Muchnik, J. (2002). Systèmes techniques, transmission et diffusion des savoir-faire : aspects méthodologiques.

14. Diawara, B. \& Ouedraogo, J-B. (2002). Les pratiques alimentaires à Ouagadougou, Burkina Faso: Céréales, légumineuses, tubercules et légumes. CNRST, CIRAD, $147 \mathrm{p}$.

15. Doebley, J. \& Iltis, H. (1980). Taxonomy of Zea (Gramineae). I. A subgeneric classification with key to taxa. Am. J. Bot. 67: 982-993.

16. DSRP (2009). " Document de relance du développement et de réduction de la pauvreté ». Côte d'Ivoire, Janvier 2009.

17. Duval, C. (2009). «Matières plastiques et environnement », édition Dunod, Paris.

18. Elwert, K. (2002). Désir de changement : transformations alimentaires au Bénin, Cotonou.

19. Engel, E. (1895). Die Lebenskosten belgischer Arbeiter familien frueher und jetzt. Ermitteltaus Familien haus halts rechnungenund vergleichend zusammen gestell, Bulletin of the International Institute of Statistics, 9, 1895, pp. 57etsuiv.

20. Escalon, H., Bossard, C. \& Beck, F. (2009). Baromètre Santé Nutrition 2008, Saint Denis.

21. FAO (1995). Le sorgho et les mils dans la nutrition humaine, collection F.A.O n 27 : alimentation et nutrition. Ed. Rome.

22. FAO (2004). Vitamin and mineral requirements in human nutrition. FAO Ed, Genève,

23. Fournier, S., Muchnik, K.J. \& Requier-Desjardins, D. (2001). Enjeux et contraintes du développement de la filière huile de palme au Bénin : une approche par les systèmes agro-alimentaires localisés, en préparation pour le colloque international «Un produit, une filière, un territoire », Université Toulouse-le-Mirail, Laboratoire Dynamiques Rurales. 
24. Gojard, S. (2000). «L'alimentation dans la prime enfance, diffusion et réception des normes de puériculture », Revue Française de Sociologie, vol $41 \mathrm{n}^{\circ}$ 3, pp. 475-512.

25. Gutierrez, M.L. (2000). Sous la direction de Maizi P, Nago MC, Hounhouigan N J. Production et commercialisation de l'afitin fon dans la région d'Abomey-Bohicon au Bénin. Un exemple d'intégration des femmes dans la filière du Néré, $124 \mathrm{p}$.

26. Guyomard, H. (2008). Boom des cours mondiaux de céréales : feu de paille ou début d'une nouvelle ère? Grain de sel, n40

27. Halbwachs, M. (1913). La classe ouvrière et les niveaux de vie. Recherches sur la hiérarchie des besoins dans les sociétés industrielles contemporaines, Paris, Alcan

28. INS (2008). «Enquête sur le niveau de vie des ménages » Institut National de la Statistique.

29. Konkobo, C. (1999). Etude des situations alimentaires des ménages et les pratiques d'alimentation dans l'espace de la restauration alimentaire: le cas de Ouagadougou. Mémoire des sociologies, option sociologie urbaine, Université de Ouagadougou, Burkina Faso. Flashs.

30. Larmet (2002). «L'organisation des achats alimentaires», Cahiers d'Economie et de Sociologie Rurales, n 63, pp. 51-84.

31. M'Biandoun, M., Theze, M. \& Abou, A. (2003). 'Maintien ou amélioration du potentiel productif des sols en région soudanosahélienne du Nord-Cameroun', in Jamin J.Y., Seiny Boukar L. Savanes africaines : des espaces enmutation, des acteurs face à de nouveaux défis. Actes du colloque, mai 2002, Garoua, Cameroun.

32. Ministère de l'agriculture (2009). «Enquête approfondie de la sécurité alimentaire : EASA $2009 »$.

33. Mitchikpe, C.E., Ategbo, E.A., Fanou, J. \& Nago, M.C. (2001). Consommation et styles de ménages urbains au Bénin.

34. Multon, J. L. (1982). Conservation des grains et graines et produits dérivés, édition Lavoisier. 576p.

35. O’deye, B.N. (1985). A propos de l'évolution des styles alimentaires à Dakar. In nourrir les villes en Afrique Sub saharienne. Paris, France, l'Harmattan, p.179-195.

36. PAM \& FAO (2008). « Système de suivi de la sécurité alimentaire, note de synthèse : round de mai 2008».

37. Paugam, S. (2002). La Société française et ses pauvres, Paris, PUF coll. Quadrige, $336 \mathrm{p}$.

38. Pierru, E. (2008). «Le crépuscule des catégories socioprofessionnelles », Revue française de science politique. 
39. Regnier, F. (2002). "Penser l'Autre en Europe: l'exotisme culinaire en France et en Allemagne », in Villain-Gandossi C. (dir.), L'Europe à la recherche de son identité, Actes des Congrès nationaux des sociétés historiques et scientifiques, Paris, Editions du CTHS, pp. 421434. WEBER F., 1998, L'honneur des jardiniers.

40. Seguy, L. (2006). Suivi évaluation et propositions de rechercheaction pour la diffusion du Semis direct sur Couverture Végétal eau Nord Cameroun, Rapport de mission du 28 Septembre au 5 octobre 2006, CIRAD/IRAD/AFD/SODECOTON, Document obtenu sur le site Cirad du réseau http://agroecologie.cirad.fr, $162 \mathrm{p}$.

41. Seignobos, C. (2005). 'Raimond C. (éd.), Garine E. (ed.), Langlois O. (éd.). Ressources Vivrières et choix alimentaires dans le bassin du lac Tchad, Colloque International Méga-Tchad (FRA), Paris : Editions IRD, Prodig., (Colloques et Séminaires), p. 527-561.

42. Silguy, C. (2009). "Histoire des hommes et de leurs déchets : du moyen âge à nos jours », édition Le cherche midi, Paris, 2009. 